\title{
Aneurisma do seio de Valsalva: análise de sete casos
}

\author{
Ricardo M. MUSTAFÁ*, Domingo M. BRAILE*, Roberto V. ARDITO*, José Luiz Verde dos SANTOS*, \\ Marcos ZAIANTCHIK*, Marcelo José Ferreira SOARES*, João Carlos Ferreira LEAL*
}

RBCCV 44205-131

\begin{abstract}
MUSTAFÁ, R. M.; BRAILE, D. M.; ARDITO, R. V.; SANTOS, J. L. V.; ZAIANTCHIK, M.; SOARES, M. J. F.; LEAL, J. C. F.; - Aneurisma do seio da Valsalva: análise de sete casos. Rev. Bras. Cir. Cardiovasc., 6(1): 45-48, 1991.

RESUMO: Os autores apresentam a experiência acumulada de janeiro de 1984 a julho de 1990, no Instituto de Moléstias Cardiovasculares (IMC), de sete casos de aneurisma do seio de Valsalva (ASV), tratados cirurgicamente com uso de retalho de pericárdio bovino (PB), ou troca valvar nos casos indicados. Cinco casos eram de origem congênita e dois estavam associados a doença reumática. Dos congênitos, quatro estavam rotos, sendo dois para o átrio direito e dois para o ventrículo direito; em um caso não havia rotura e se projetava para o ventrículo esquerdo. Dos dois casos associados a doença reumática, um estava roto para o ventrículo direito e o outro apresentava protrusão do saco aneurismático para o ventrículo esquerdo e eversão da válvula aórtica correspondente. Foi usado retalho de PB para dar sustentação à sutura nos casos de ASV congênitos; destes, um evoluiu com deiscência da sutura aos sete meses e aos seis anos, sendo reoperado nas duas ocasiōes. Nos casos associados a doença reumática, foi realizada a substituição valvar com prótese biológica PB IMC; dos dois pacientes, um foi a óbito, em decorrência de acidente vascular cerebral, no 15: dia de pós-operatório. Conclui-se que, quando não há alteraçōes secundárias nas válvulas (ex.: calcificação, endocardite, etc.), a restauraçāo é possível e desejada, com boa evoluçāo quando se usa retralho de pericárdio bovino.
\end{abstract}

DESCRITORES: aneurismas do seio de Valsalva, cirurgia.

\section{INTRODUÇÃO}

Os aneurismas do seio de Valsalva (ASV) constituem afecção pouco comum, de origem congênita ou adquirida, apresentando-se comumente rotos e, algumas vezes, sem rotura e com pouca expressão clínica ${ }^{4}$. 7. 11. Na maioria das vezes, quando rotos, a terapêutica cirúrgica se impōe ${ }^{2}$, haja visto que se expressam de forma clínica grave. A técnica adotada está sujeita a variaçōes, dependendo da apresentação da doença no que se refere à integridade das válvulas e da parede aórtica, e de lesōes associadas. A simples sutura da base do $\mathrm{ASV}^{3}{ }^{3}{ }^{19}$, excisão e sutura do aneurisma com retalho sintético ${ }^{14}$, ou a ressecção e substituição valvar ${ }^{2}$. ${ }^{13}$, são algumas das medidas recomendadas.
Os autores analisam sete casos de ASV, enfatizando seu tratamento cirúrgico e avaliando a evolução dos pacientes com as técnicas empregadas. Preconizam a utilização de retalho de pericárdio bovino para melhor reconstituição da integridade anátomo-funcional aórtica.

\section{CASUÍSTICA E MÉTODOS}

São analisados sete casos de ASV tratados cirurgicamente no IMC, no periodo de janeiro de 1984 a julho de 1990 , submetidos a nove procedimentos cirúrgicos. A idade variou de 8 a 41 anos, com média de 25 , sendo quatro deles homens.

Trabalho realizado no Instituto de Moléstias Cardiovasculares de Săo José do Rio Preto, SP, Brasil.

Recebido para publicaçăo em 15 de fevereiro de 1991.

* Do Instituto de Moléstias Cardiovasculares de Sảo José do Rio Preto.

Endereço para separatas: Ricardo M. Mustafá. Rua Castelo D'Água, 3030. 
MUSTAFÁ, R. M.; BRAILE, D. M.; ARDITO, R. V.; SANTOS, J. L. V.; ZAIANTCHIK, M.; SOARES, M. J. F.; LEAL, J. C. F.; Aneurisma do seio da Valsalva: análise de sete casos. Rev. Bras. Cir. Cardiovasc., 6(1): 45-48, 1991.

Todos os pacientes foram submetidos pelo menos a estudo radiológico, eletrocardiográfico, ecocardiográfico e angiográfico, além dos exames clínico e laboratorial.

A técnica cirúrgica utilizada consistiu em abertura do tórax através de esternotomia mediana e instalação do sistema de circulação extracorpórea através de canulação da aorta ascendente e do átrio direito (quando as cavidades direitas não estavam acometidas) ou seletiva das cavas (quando foi necessária a atriotomia). A proteção miocárdica utilizada foi a cardioplegia cristalóide hiperpotassêmica e hipotérmica até 1987 (cinco procedimentos), e cardioplegia sangüínea hiperpotassêmica e hipotérmica ou normotérmica após esse período (quatro procedimentos).

Dos casos analisados, cinco eram ASV de origem congênita e dois ASV associados a doença reumática. Destes, dois estavam assintomáticos e foram diagnosticados por achado de sopro sístolo-diastólico; dois outros apresentavam queixa de dispnéia e palpitação com duração de menos de um mês, um dos quais apresentou, também, precordialgia atípica; outros dois tinham insuficiência cardíaca congestiva, um com três anos de evolução e o outro desde a infância (associado a comunicação interventricular - $\mathrm{CIV}$ ). Um paciente apresentava pneumonias de repetição.

À ausculta, encontrou-se sopro sístolo-diastólico em cinco ocasiōes, e em dois apenas sopro sistólico. Variavam na localização e intensidade de acordo com a associação de outras lesōes e, principalmente da comunicação cavitária estabelecida.

O ECG e a radiografia de tórax mostravam sobrecarga das câmaras cardiacas acometidas (Tabela 1). O diagnóstico de certeza foi feito pelos estudos eco e angiocardiográfico que possitilitaram a realização do diagnóstico de ASV, observando-se a protrusão aneurismática e presença ou não de rotura (Tabela 1), bem como as lesões associadas (Tabela 2).

TABELA 1 CORRELAÇÃO DA PROTRUSÃO ANEURISMATTICA COM A CAMARA CARDIACA ACOMETIDA E A PRESENÇA OU NÄO DE SHUNT.

\begin{tabular}{lccc}
\hline SV acometido & $\begin{array}{c}\text { Cámara } \\
\text { acometida }\end{array}$ & $\begin{array}{c}N^{0} \text { de } \\
\text { casos }\end{array}$ & $\begin{array}{c}\text { Presença } \\
\text { de rotura }\end{array}$ \\
\hline Seio não coronariano & AD & 2 & Sim \\
& VD & 1 & Sim \\
Seio coronariano direito & VE & 1 & Não \\
Seio coronariano esquerdo & VE & $2^{*}$ & Sim \\
\hline
\end{tabular}

- Presença de um caso de aneurisma do seio de Valsalva associado a doença reumática.

$A D=$ átrio direito; $V D=$ ventrículo direito; $V E$ = ventrículo esquerdo; SV = seio de Valsalva.
TABELA 2

DOENCYAS CARDIACAS CONGENITAS ASSOCIADAS

\begin{tabular}{lc}
\hline Doenças & Ocorrência \\
\hline Valva aórtica bivalvulada & 2 \\
CIV & 1 \\
Coarctaçāo aórtica, PCA, túnel subaórtico & 1 \\
Insuficiência mitral & 1 \\
\hline
\end{tabular}

$\mathrm{CIV}=$ comunicação interventricular; $\mathrm{PCA}=$ persistência do canal arterial.

Em dois casos, foi realizada a troca valvar com prótese de pericárdio bovino (PB) IMC, em decorrência de lesão das válvulas por doença reumática. Nos demais procedimentos, foram usados retalhos de pericárdio bovino para reforço na base da sutura e/ou correção de lesão associada.

\section{RESULTADOS}

Dos sete pacientes analisados, observou-se um óbito conseqüente e acidente vascular cerebral no $15^{\circ}$ dia de pós-operatório. Tal paciente foi submetido a troca da prótese valvular aórtica no pós-operatório imediato,
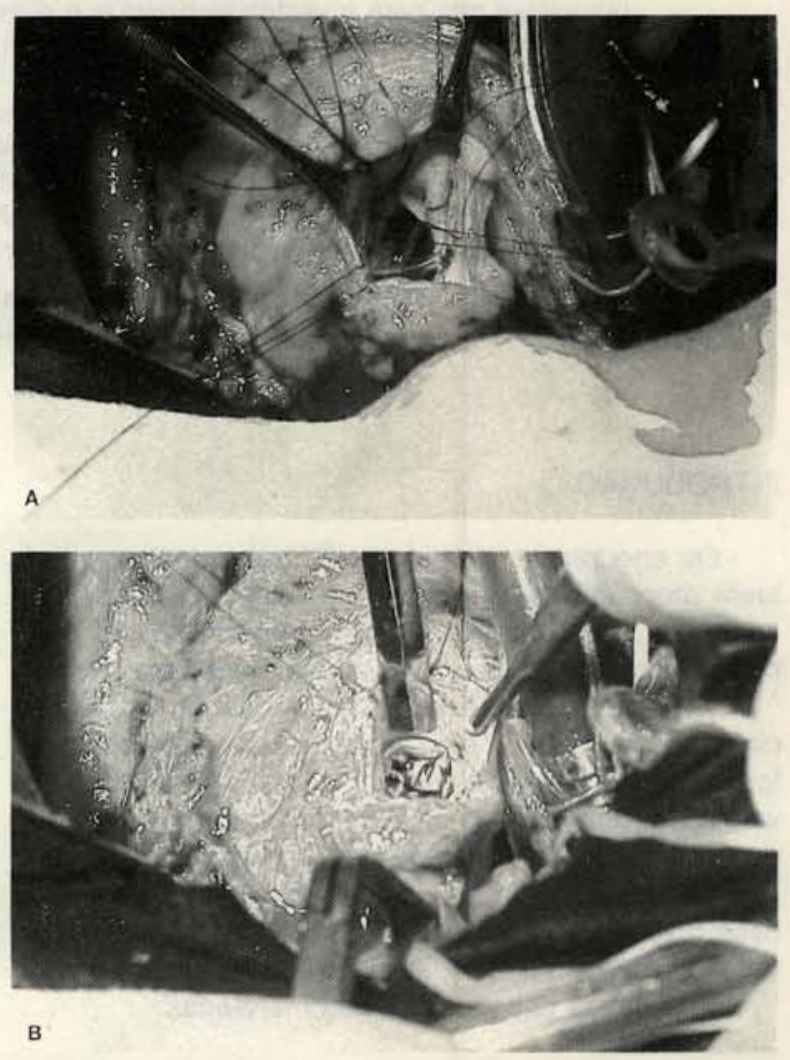

Fig. 1 - A: Aneurisma do seio de Valsalva náo coronariano roto para átrio direito. Observa-se um probe introduzido pelo átrio direito, visto pela aortotomia. B: Após a correção cirúrgica com retalhos de PB, mostrando a integridade anatómica valvar preservada. 
MUSTAFÁ, R. M.; BRAILE, D. M.; ARDITO, R. V.; SANTOS, J. L. V.; ZAIANTCHIK, M.; SOARES, M. J. F.; LEAL, J. C. F.; Aneurisma do seio da Valsalva: análise de sete casos. Rev. Bras. Cir. Cardiovasc., 6(1): 45-48, 1991.

em decorrência de vazamento paravalvular. Em outro caso, houve recorrência de rotura, por duas vezes aos sete mesese seis anos após a correção inicial, sendo a primeira no mesmo local da cirurgia anterior e a segunda ao lado. Nas duas ocasiōes, utilizou-se duplo retalho, na face aórtica e atrial, para o fechamento da comunicação (Figura 1).

Todos os pacientes evoluíram em classe funcional I (NYHA) no seguimento de um a seis anos $(M=4)$.

\section{DISCUSSÃO}

Os aneurismas do seio de Valsalva são lesōes pouco freqüentes, tendo-se observado, no entanto, um incremento em seu diagnóstico com a melhoria dos métodos propedêuticos. MEYER et alii ${ }^{13}$ observaram uma incidência de $0,43 \%$ de ASV em 10370 cirurgias cardiacas. Em nossa avaliação, obtivemos um índice de 0,21\%.

Quanto à etiologia, controvérsias persistem até os dias atuais: a) adquirida: a endocardite infecciosa representou, em artigo de revisāo, a causa mais freqüente 1 , ao contrário da nossa experiência. A doença reumática não é incomum ${ }^{13}$, ocorrendo em dois $(28,6 \%)$ de nossos pacientes, porém não pudemos estabelecer a precedência da origem desta em relação ao do ASV. b) Congênitos: para EDWARDS et alii ${ }^{5}$ e ELIOT et alii ${ }^{6}$, a clivagem entre a camada média e o esqueleto fibroso do coração poderia predispor os seios aórticos a uma fragilidade que formaria saculaçōes aneurismáticas, frente a fatores precipitantes, tal como a elevada pressão na raíz da aorta. KWITTKEN et alii ${ }^{12}$ acreditam que estas alteraçōes sāo possiveis de se encontrar, desde que haja a dilatação aneurismática. SAKAKIBARA \& KONNO ${ }^{15}$ responsabilizaram a CIV como causa do ASV, corroborando achados anteriores 9,10 .

Quanto à localização, encontramos o seio não coronariano como o mais acometido $(57,1 \%)$, seguido do seio coronariano direito (28,6\%). Entretanto, alguns autores $^{2,14}$ referem-se ao aneurisma do seio coronariano direito como o mais freqüente, variando entre $67,2 \%$ e $85 \%$. Aneurisma do seio coronariano esquerdo é o mais raro.

A rotura é a complicação mais comum do ASV, sendo que a sintomatologia apresentada é um reflexo da comunicação cavitária estabelecida e da falência hemodinâmica ${ }^{2}$. Poucos ${ }^{8}$ são os relatos de ASV em rotura, devido a pobre expressão clínica ${ }^{4}, 7,11$. Apenas dois dos nossos casos não estavam rotos, e o diagnóstico foi realizado em decorrência de insuficiência aórtica (por eversão da válvula) em um caso, e por lesão associada em outro (coarctação da aorta, persistência do canal arterial, túnel subaórtico). O seio não coronariano rompeu para o AD duas vezes, VD uma vez, estando protruso e não roto para VE uma vez. Há referências, na literatura, de que o ASV rompe para $A D$, sendo raro para VD ${ }^{16}$ (2 a $5 \%$ ) e ainda mais raro para VE ${ }^{18}$. BURAKOVSKY et alii ${ }^{2}$ relataram indices de $85 \%$ a $95 \%$ para rompimento do seio direito para VD e de $5 \%$ a $15 \%$ para AD. No entanto, observou-se, neste trabalho, um índice de $28,5 \%$ de rompimento do seio direito para o VD.

Os princípios básicos para a correção de tais anomalias foram enfatizados por NOWICKI et alii ${ }^{14}$, segundo os quais deveria se almejar fechamento adequado das fístulas coexistentes, bem como seria recomendável a correção de quaisquer anormalidades cardiacas associadas.

A excisão da saculação aneurismática e correção com retalho de PB é proposta. Os autores acreditam que isso proporciona possibilidade de reconstrução das válvulas aórticas. Entretanto, às vezes é necessária a troca valvar, como aconteceu por duas vezes, devido a lesão das válvulas aórticas, por doença reumática cicatrizada, impossibilitando a plástica.

RBCCV 44205-131

MUSTAFÁ, R. M.; BRAILE, D. M.; ARDITO, R. V.; SANTOS, J. L. V.; ZAIANTCHIK, M.; SOARES, M. J. F.; LEAL, J. C. F.; - Aneurysm of the sinus of Valsalva: analysis of seven cases. Rev. Bras. Cir. Cardiovasc., 6(1): 45-48, 1991.

ABSTRACT: The authors present their experience on seven cases of Valsalva-sinus aneurysm (VSA) at the Instituto de Moléstias Cardiovasculares (IMC), from January 1984 to July 1990 , treated surgically using bovine pericardium patch (PB-IMC) or valve replacement when indicated. Five cases were congenital and two were related to rheumatic disease. In two of the congenital cases the aneurysms were leaking into the right atrium, two were leaking into the right ventricle. And the remaining one was not leaking but was projected into the left ventricle. One of the congenital cases was leaking into the right ventricle and the other presented protrusion of the aneurysmatic sac into the left ventricle and eversion of the corresponding aortic leaflets. A bovine pericardium patch was used to give support for he sutures in the congenital ASV cases; for the cases related to rheumatic disease a valve replacement was performed using an IMC-PB biologic valve. From the congenital VSA patients, one had deiscence of suture at seven months and at six years, and was submitted to reoperation in both occasions. In the related to rheumatic disease group one died due to stroke on the $15^{\text {th }}$ postoperative day. The use of PB patch proved satisfactory in this treatment, providing good support for the suture.

DESCRIPTORS: aneurysms of the sinus of Valsalva, surgery. 
MUSTAFÁ, R. M.; BRAILE, D. M.; ARDITO, R. V.; SANTOS, J. L. V.; ZAIANTCHIK, M.; SOARES, M. J. F.; LEAL, J. C. F.; Aneurisma do seio da Valsalva: análise de sete casos. Rev. Bras. Cir. Cardiovasc., 6(1): 45-48, 1991.

\section{REFERÊNCIAS BIBLIOGRÁFICAS}

1 BUCHBINDER, N. A. \& ROBERTS, W. C. - Left-sided valvular active infective endocarditis: a study of fortyfive necropsy patients. Am. J. Med., 53: 20-35, 1972.

2 BURAKOVSKY, V. I.; PODSOLKOV, U. P.; SABIROW, B. N.; NASEDKINA, M. A.; ALEKIAN, B. G.; DVINYANINOVA, N. B. - Ruptured congenital aneurysm of the sinus of Valsalva. J. Thorac. Cardiovasc. Surg., 95: $836-841,1988$.

3 De BAKEY, M. E.; DIETRRICH, E. B.; LIDDICOAT, J. E.; KINARD, S. A.; GORRETT, H. E. - Abnormalities of the sinuses of Valsalva experience with 35 patients. J. Thorac. Cardiovasc. Surg., 54: 312-332, 1967.

4 DURAS, P. F. - Heart block with aneurysm of the aortic sinus. Br. Heart J., 6: 61-65, 1944.

5 EDWARDS, J. E.; BURCHELL, H. B.; CHRISTENSEN, N. A. - Specimen exhibiting the essencial lesion in aneurysm of the aortic sinus. Proc. Staff Meet. Mayo Clin., 31: 407-412, 1956.

6 ELIOT, R. S.; WOLBRINK, A.; EDWARDS, J. E. - Congenital aneurysm of the left aortic sinus: a rare lesion and a rare cause of coronary insufficiency. Circulation, 28: 951-956, 1963.

7 FALHOLT, W. \& THOMSEN, G. - Congenital aneurysm of the right sinus of Valsalva, diagnosed by aortography. Circulation, 8: 549-553, 1953.

8 FISHBEIN, M. C.; OBMA, R.; ROBERTS, W. C. - Unruptured sinus of Valsalva aneurysm. Am. J. Cardiol., 35: 918-922, 1975

9 JONES, A. M. \& LANGLEY, F. A. - Aortic sinus aneurysms. Br. Heart J, 11: 325-341, 1949.

10 KAKOS, G. S.; KILMAN, J. W.; WILLIAMS, T. E.; HOSIER, D. M. - Diagnosis and management of sinus of Valsalva aneurysm in children. Ann. Thorac. Surg., 17: 474-478, 1974.

11 KERBER, R. E.; RIDGES, J. D.; KRISS, J.P.; SILVERMAN, J. F.; ANDERSON, E. T.; HARRISON, D. C. - Unrup- tured aneurysm of the sinus of Valsalva producing right ventricular outflow obstruction. $A m \mathrm{~J}$. Med., 53: 775-783, 1972.

12 KWITTKEN, J.; CHRISTOPOULOS, P.; DUA, N. K.; BRUNO, M. S. - Congenital and acquired aortic sinus aneurysm: a case report of each with histologic study. Arch. Intern. Med., 115: 648-691, 1965.

13 MEYER, J.; WUKASCH, D. C.; HALLMAN, G. L.; COOLEY, D. A. - Aneurysm and fistula of the sinus of Valsalva clinical considerations and surgical treatment in 45 patients. Ann. Thorac. Surg., 19: 170-179, 1975.

14 NOWICKI, E. R.; ABERDEEN, E.; FRIEDMAN, S.; RASHKING, W. J. - Congenital left aortic sinus: left ventricle fistula and review of aortocardiac fistulas. Ann. Thorac. Surg., 23: 378-388, 1977.

15 SAKAKIBARA, S.; KONNO, S. - Congenital aneurysm of the sinus of Valsalva associated with ventricular septal defect. Am Heart J., 75: 595-603, 1968.

16 SAWYERS, J. L.; ADAMS, J. E.; SCOTT, W. - Surgical treatment for aneurysm of the aortic sinuses with aorticoatrial fistula. Surgery, 41: 26-42, 1957.

17 SHUMACKER, H. B. - Aneurysms of the aortic sinuses of Valsalva due to bacterial endocarditis, with special reference to their operative management. $J$. Thorac. Cardiovasc. Surg., 63: 896-902, 1972.

18 SHUMACKER, H. B. \& JUDSON, W. F. - Rupture of aneurysm of sinus of Valsalva into left ventricle and its operative repair. J. Thorac. Cardiovasc. Surg., 45: 650-656, 1963.

19 SPENCER, F. C.; BLAKE, H. A.; BAHNSON, H. T. - Surgical repair ruptured aneurysm of sinus of Valsalva in two patients. Ann. Surg., 152: 963-968, 1960.

AGRADECIMENTO: Este trabalho teve a colaboração da Divisão de Pesquisas e Publicações (DDP) do IMC - Biomédica, do Centro de Processamento de Dados (CPD) do IMC, e do Clube do Coração (CDC) dos Residentes de Cirurgia Cardíaca, IMC, São José do Rio Preto, SP. 